\title{
BMI Is an Independent Preoperative Predictor of Intraoperative Transfusion and Postoperative Chest-Tube Output
}

\author{
Heather R. Nolan, MD ${ }^{1}$ Daniel L. Davenport, $\mathrm{PhD}^{2}$ \\ ${ }^{1}$ Department of Surgery, Mercer University School of Medicine, \\ Macon, Georgia \\ 2 Department of Surgery, University of Kentucky College of Medicine, \\ Lexington, Kentucky \\ ${ }^{3}$ Division of Cardiothoracic Surgery, Department of Surgery, \\ University of Kentucky College of Medicine, Lexington, Kentucky \\ Int J Angiol 2013;22:31-36.
}

Chandrashekhar Ramaiah, $\mathrm{MD}^{3}$

\begin{abstract}
Address for correspondence Chandrashekhar Ramaiah, MD, Division of Cardiothoracic Surgery, Department of Surgery, University of Kentucky, 740 South Limestone, Room A301, Lexington, KY 40536-0284 (e-mail: crama01@uky.edu).
\end{abstract}

\begin{abstract}
Background An increasing obese population in the United States focuses attention on perioperative management of obese and overweight patients.

Objective We sought to determine if obesity, determined by body mass index (BMI), was a preoperative indicator of bleeding in coronary artery bypass graft (CABG) surgery as measured by intraoperative packed red blood cell transfusion frequency and 24-hour chest-tube output amount.

Methods A retrospective chart review examined 290 consecutive patients undergoing single-surgeon off-pump or on-pump CABG surgery between November 2003 and April 2009. Preoperative variables of age, gender, hematocrit, platelet count, and BMI, chest tube output during the immediate 24-hour postoperative period, and the type of procedure (on-pump vs. off-pump) were analyzed. Logistic regression analysis was used to evaluate the likelihood of intraoperative transfusion. Linear regression analysis was used to evaluate 24-hour chest-tube output.

\section{Keywords}

- obesity

- BMI

- CABG

- intraoperative transfusion

- chest-tube output

Results Preoperative variables that significantly increased the likelihood of intraoperative transfusions were older age and low hematocrit; a significant decrease in likelihood was found with male gender, overweight BMI, and off-pump procedures. Preoperative variables that significantly increased 24-hour chest-tube output were low hematocrit, high hematocrit, and low platelets while a significant decrease in output was seen with overweight BMI and obese BMI.

Conclusion Overweight and obese BMI are significant independent predictors of decreased intraoperative transfusion and decreased postoperative blood loss.
\end{abstract}

Obesity is a global health care concern with rising trends across the world and the United States. In 2008, the World Health Organization found 1.5 billion adults were overweight with a staggering 200 million men and almost 300 million women being obese. ${ }^{1}$ In the United States, 60 to $70 \%$ of adults are overweight or obese, and the prevalence of obesity continues to rise with nearly one in three adults classified as obese. ${ }^{2,3}$ This patient population has become an area of focus as obesity can affect a wide variety of clinical parameters in addition to being associated with increased risk of heart disease, stroke, and type 2 diabetes. ${ }^{2,3}$ In addition, in cardiac surgery, although there seems to be no effect on mortality, controversy exists regarding increased morbidity in obese patients undergoing heart operations. ${ }^{4-6}$ Thus, specific variables are being assessed to determine the effect of obesity on individual clinical parameters in cardiac 
procedures. In this study, we examined coronary artery bypass graft (CABG) surgery and how obesity impacted intraoperative transfusion requirements and postoperative bleeding.

Understanding intraoperative transfusion requirements will streamline blood bank usage and facilitate patient-specific interventions. Preoperative parameters that predict usage are varied, with clinical assessment tools already in place for many operative indications such as trauma patients requiring massive transfusion. ${ }^{7}$ Cardiac surgery in particular routinely utilizes red blood cells, and research is ongoing for evaluating which patients will need blood and how much. ${ }^{8-10}$

Just as with transfusions, a tool to evaluate patients preoperatively for their estimated blood loss postoperatively will give better patient-specific care. Research evaluating which factors can predict postoperative blood loss exists ${ }^{11,12}$; however, the large proportion of existing literature evaluates blood loss based on transfusion requirements or looks purely at rate of reoperation for bleeding. In our study, we diverged from this practice and utilized chest-tube output as a direct means of measuring blood loss at the surgical site.

A previous study by our group found a decrease in both transfusions and chest-tube output with increasing obesity in CABG surgery. ${ }^{13}$ We wanted to further evaluate this relationship, however, with the goal of our current study being to utilize a much larger cohort and multivariable analyses. These new methods allowed us to ascertain the significance of BMI as an independent preoperative indicator of intraoperative transfusion and postoperative blood loss.

\section{Methods}

Institutional Review Board approval was obtained prior to data collection for this study. Data were collected via a retrospective chart review. Data were collected from consecutive patients who received isolated CABG surgery (on- or off-pump), performed by a single surgeon at the University of Kentucky Medical Center between November 2003 and April 2009. A total of 338 charts were reviewed with 290 patients meeting our inclusion criteria included in the analysis (-Table 1). Patients undergoing simultaneous valve procedures, aneurysms, or patent foramen ovale repair were excluded. Patients whose charts were missing specified preoperative variables including preoperative hematocrit and platelet levels were also excluded.

BMI was calculated by dividing the individual patient's weight in kilograms by the individual patient's height in meters squared. Following American Heart Association and the World Health Organization guidelines, patients were divided into groups based on BMI and included normal weight (BMI $<25$ ), overweight (BMI 25 to 29), and obese $(\mathrm{BMI} \geq 30){ }^{1,14}$ Hematocrit was categorized as normal (range of 35.1 to $44.2 \%$ ), low ( $<35 \%$ ), and high ( $>44.3 \%$ ), with the normal hematocrit group used as reference. Platelet count was categorized as normal or low $\left(<150 \times 10^{3}\right.$ per milliliter $)$ with a normal platelet count used as reference. Packed red blood cell transfusions were assessed by the total number of transfusions received intra-operatively. Transfusion triggers
Table 1 Patient demographics relative to each analyzed preoperative variables, $n=290$

\begin{tabular}{|l|l|}
\hline Variable & Incidence \\
\hline Age & Mean 61.9 \pm SD 10.5 \\
\hline Male & $83.8 \%(n=243)$ \\
\hline Normal hematocrit & $64.5 \%(n=187)$ \\
\hline Low hematocrit & $17.9 \%(n=52)$ \\
\hline High hematocrit & $17.6 \%(n=51)$ \\
\hline Normal platelets & $91.7 \%(n=266)$ \\
\hline Low platelets & $8.3 \%(n=24)$ \\
\hline Normal BMI & $19.3 \%(n=56)$ \\
\hline Overweight BMI & $43.5 \%(n=126)$ \\
\hline Obese BMI & $37.2 \%(n=108)$ \\
\hline On-pump CABG & $84.8 \%(n=246)$ \\
\hline Off-pump CABG & $15.2 \%(n=44)$ \\
\hline
\end{tabular}

Abbreviations: SD, standard deviation; BMI, body mass index; CABG, coronary artery bypass graft.

included patients with a hematocrit of less than $21 \%$ or hemoglobin less than $7 \mathrm{~g} / \mathrm{dL}$ or elderly/actively bleeding patients with a hematocrit of less than $24 \%$ or a hemoglobin less than $8 \mathrm{~g} / \mathrm{dL}$. Final transfusion decisions were made intraoperatively by the surgeon. Chest-tube output was calculated using the amount in milliliters collected from the chest-tube draining the surgical site during the immediate 24-hour postoperative period. Chest-tube placement was standard of care for the procedure, and values were recorded as total amount regardless of number of chest tubes placed.

Two separate multivariable regression analyses were done. Logistic regression evaluated the likelihood of intraoperative transfusion related to age, gender, BMI, preoperative hematocrit, preoperative platelet count, and procedure type (offpump vs. on-pump). Linear regression evaluated the natural logarithm of 24-hour chest-tube output related to the same variables. In both analyses, the significance level was set at $p<0.05$. SPSS ${ }^{\mathrm{TM}}$ Version 19 software was used for all statistical analysis (SPSS, Chicago, IL).

\section{Results}

Among these 290 patients, the mean age was 61.9 years (SD of 10.5 years), and the majority of patients were male ( $n=243$, $83.8 \%$ ). The normal hematocrit group accounted for $64.5 \%$ of the cohort $(n=187)$, whereas the low hematocrit group accounted for $17.9 \%$ ( $n=52$ ), and the high hematocrit group was similarly $17.6 \%(n=51)$. The normal platelet group accounted for $91.7 \%(n=266)$ of the cohort and the low platelet group $8.3 \%(n=24)$. The normal weight BMI group accounted for $19.3 \%$ of the cohort $(n=56)$, the overweight group for $43.5 \%(n=126)$, and the obese for $37.2 \%(n=108)$. On-pump procedures accounted for $84.8 \%$ of the cohort ( $n=246)$, whereas $15.2 \%$ had off-pump procedures $(n=44)$. 
Table 2 Logistic regression analysis of intraoperative transfusion

\begin{tabular}{|l|l|l|l|}
\hline \multicolumn{5}{|c|}{ Incidence of intraoperative transfusions $(\boldsymbol{n}=85 ; 29.3 \%)$} \\
\hline Variable & Odds ratio & $95 \%$ Confidence interval & $P$-value \\
\hline Age & 1.045 & $1.013-1.077$ & 0.005 \\
\hline Male & 0.138 & $0.062-0.305$ & $<0.001$ \\
\hline Low hematocrit & 5.473 & $2.591-11.562$ & $<0.001$ \\
\hline High hematocrit & 0.373 & $0.128-1.093$ & 0.072 \\
\hline Low platelets & 1.730 & $0.597-5.013$ & 0.312 \\
\hline Overweight BMl & 0.359 & $0.165-0.783$ & 0.010 \\
\hline Obese BMl & 0.327 & $0.145-0.739$ & 0.007 \\
\hline Off-pump & 0.236 & $0.084-0.668$ & 0.007 \\
\hline
\end{tabular}

Abbreviations: BMI, body mass index; $C A B G$, coronary artery bypass graft.

Eighty-five patients received intraoperative transfusions (29.3\%). Preoperative variables that significantly increased the likelihood of intraoperative transfusions were older age and low hematocrit, whereas male gender, overweight and obese BMI groups, and off-pump procedures decreased the likelihood of intraoperative transfusions (-Table 2). Low platelet count and high hematocrit were not significant contributors to intraoperative transfusion.

The median 24-hour chest-tube output amount was $823 \mathrm{~mL}$ with an interquartile range of 631 to $1,070 \mathrm{~mL}$. Preoperative variables that significantly increased 24-hour chest-tube output were low hematocrit, high hematocrit, and low platelets whereas overweight and obese BMI significantly decreased 24-hour chest-tube output (-Table 3). Due to the natural log transformation, the regression coefficients should be interpreted as the percent change in output per unit change of the predictor variable. Male gender, age, and offpump procedure were not significant predictors of 24-hour chest-tube output.

Our results are in line with several studies that have shown a lower BMI to be related to increased transfusion requirements. ${ }^{15-18}$ Additional studies have had similar results using BSA or weight as the variable and noted an increase in transfusion with a lower BSA or lower weight. ${ }^{10,19-21}$ There is difficulty correlating many of these results with our study for several reasons. When BMI is used as a variable it is often defined as simply low BMI or as obese. ${ }^{15}$ This does not allow for separate analysis of BMI categories beyond the extreme ranges of the scale. The advantage to fully stratifying the classifications is that it leads to a better understanding of more moderate changes in weight. The fact that we saw significance in our results with overweight and obese BMI points to the idea that excess body weight is a contributing factor to transfusion requirements and it is not merely just the extremes of the malnourished and the morbidly obese that cause variances from normal. One study that did analyze BMI groups was Reeves et al who examined underweight, normal, overweight, obese, and severely obese patient populations undergoing CABG. ${ }^{18}$ Reeves et al found that the overweight, obese, and morbidly obese had a linearly decreasing odds ratio relevant to red blood cell transfusion. ${ }^{18}$ The difficulty with the Reeves study, however, is a complication in many additional studies. These studies record transfusions as an occurrence and do not further categorize transfusions based on broad timing of transfusion (i.e., intraoperative or postoperative). Without such characterization it

Table 3 Linear regression analysis of 24-hour chest-tube output

\begin{tabular}{|l|l|l|l|}
\hline \multicolumn{5}{|c|}{ 24-hour chest-tube output $^{\text {a }}$} \\
\hline Variable & Regression coefficient & 95\% Confidence interval & $P$-value \\
\hline Age & 1.044 & $0.999-1.009$ & 0.080 \\
\hline Male & 0.994 & $0.865-1.141$ & 0.930 \\
\hline Low hematocrit & 1.152 & $1.016-1.308$ & 0.028 \\
\hline High hematocrit & 1.153 & $1.008-1.319$ & 0.038 \\
\hline Low platelets & 1.313 & $1.132-1.523$ & $<0.001$ \\
\hline Overweight BMI & 0.836 & $0.738-0.947$ & 0.005 \\
\hline Obese BMI & 0.791 & $0.691-0.905$ & 0.001 \\
\hline Off-pump & 0.987 & $0.857-1.136$ & 0.852 \\
\hline
\end{tabular}

${ }^{\mathrm{a}}$ Median output $=823 \mathrm{~mL}$.

Abbreviations: BMI, body mass index; CABG, coronary artery bypass graft. 
is difficult to assess and estimate blood product requirements during the CABG procedure. This is further proved by the results of our study in which the variables relating to postoperative bleeding are not the same as those that predict intraoperative blood product needs. Classifying transfusions based on time frame can help the surgeon and blood bank to predict the amount of blood product needed in a timelier manner; specifically, predicting intra-operative blood usage can better define the product that needs to be in the operating room at the time of the procedure. Our results show that intraoperative blood transfusions requirements are decreased for the overweight and obese BMI populations.

\section{Discussion}

Our results demonstrating BMI is a significant preoperative indicator of decreased chest-tube output and thus decreased postoperative bleeding correlates well with other similar studies. It is important to note that chest-tube output is only a surrogate for surgical site bleeding as other factors can contribute to drainage amount, including amount of fluid left in the pleural cavity from irrigation or intraoperative blood loss for example. There is evidence, however, that chest-tube site location does not affect output which allows us to subtract that variable from our results as our analysis did not ascertain location of chest-tubes. ${ }^{22}$ As mentioned earlier, historically studies have evaluated postoperative bleeding based either on required transfusions or reoperation for bleeding. Several studies evaluating reoperation for bleeding have had similar results to our findings with a decrease in reoperation in the obese patient population..$^{4,23-25}$ Interestingly, Alam et al examined postoperative bleeding and reoperation for bleeding and found obesity was associated with a decrease in both parameters ${ }^{23}$; however, the method by which postoperative bleeding was measured was not included in the publication. Again the closest study assimilating our results is that by Reeves et $\mathrm{al}^{18}$ who examined not only reoperation for bleeding but also blood loss as evaluated by chest-tube output. Their results showed that overweight, obese, and severely obese populations appeared to be protected against reoperation and chest-tube output greater than $1,000 \mathrm{~mL}$. In this output category the odds ratio was linear; however, further analysis of amounts less than 1,000 $\mathrm{mL}$ was not discussed. Yet, utilizing the greater than 1,000 $\mathrm{mL}$ parameter is still appropriate and applicable to our findings as Wynne et al found that mean total chest-tube output following CABG is roughly $1,300 \mathrm{~mL}^{26}$ Correlating these findings with the results of our study, our results further show that overweight and obese patient populations show a decreased percentage change in chest-tube output in the 24hour postoperative period indicating that increasing BMI is protective against postoperative surgical site blood loss.

Limitations of this study revolve mostly around it being a retrospective study as opposed to a prospective study. With a retrospective approach, there were several parameters that were unknown or not well documented and thus were unable to be included in this analysis. Such parameters include medications such as aspirin and other prescriptions affecting the coagulation cascade, which have proven to affect the amount of chest-tube output and transfusion requirements of CABG patients. $^{27}$ In addition, preoperative percutaneous intervention and specific operative variables such as cardiopulmonary bypass circuit type that could affect transfusions and bleeding were not evaluated. ${ }^{28}$

The results from our study indicate that indeed obesity as measured by BMI has a significant effect on intraoperative transfusion and postoperative bleeding. Our results are unique in that we evaluate transfusions requirements based on their relation to the procedure itself and that we define and stratify obesity based on BMI categories. In addition, our results are one of the few that utilizes surgical site drainage as a measure of postoperative bleeding and found that the preoperative variables affecting this parameter are not the same as those that would require transfusion in the operative time period. As such, we believe BMI to be an important variable in preoperative patient assessment. Further research will elucidate how to incorporate BMI into a predictive model that will give the surgeon a preoperative prediction of patient-specific coagulation. As a variable, BMI is easy to ascertain and does not require additional costs. The only potential obstacles to its collection are emergency situations in which these data points are not able to be collected. With respect, an urgent/emergent situation is in itself a separate entity and as such has shown to be strongly associated with increased transfusion rates. ${ }^{29}$ In addition to the role BMI will play in predictive tools, further research is also necessary at the molecular level to understand the etiology behind BMI's effect on coagulation. Regardless of where this research leads, this study proves that it is important at the clinical level to take into account a patient's BMI when preparing for the perioperative sequelae of CABG surgery.

\section{Conclusion}

Overweight and obese BMI is a significant independent predictor of decreased intraoperative transfusion and decreased postoperative blood loss in CABG surgery patients. These values were significant even when adjusted for low preoperative hematocrit and platelets and on-pump versus off-pump procedure type.

\section{References}

1 World Health Organization [Internet]. Obesity and overweight. Fact sheet no. 311. May 2012. http://www.who.int/mediacentre/ factsheets/fs311/en/index.html. Accessed July 15, 2012

2 American Heart Association [Internet]. Obesity information. Updated May 5, 2011. http://www.heart.org/HEARTORG/GettingHealthy/WeightManagement/Obesity/Obesity-Information_UCM_307908_Article.jsp\#.T1pFH3qi2So. Accessed July 15, 2012

3 Centers for Disease Control and Prevention [Internet]. Adult obesity facts. http://www.cdc.gov/obesity/data/adult.html. Accessed July 15, 2012

4 Engel AM, McDonough S, Smith JM. Does an obese body mass index affect hospital outcomes after coronary artery bypass graft surgery? Ann Thorac Surg 2009;88(6):1793-1800 
5 Yap CH, Zimmet A, Mohajeri M, Yii M. Effect of obesity on early morbidity and mortality following cardiac surgery. Heart Lung Circ 2007;16(1):31-36

6 Kuduvalli M, Grayson AD, Oo AY, Fabri BM, Rashid A. Risk of morbidity and in-hospital mortality in obese patients undergoing coronary artery bypass surgery. Eur J Cardiothorac Surg 2002;22 (5):787-793

7 Nunez TC, Voskresensky IV, Dossett LA, Shinall R, Dutton WD, Cotton BA. Early prediction of massive transfusion in trauma: simple as $A B C$ (assessment of blood consumption)? J Trauma 2009;66(2):346-352

8 Cobain TJ, Vamvakas EC, Wells A, Titlestad K. A survey of the demographics of blood use. Transfus Med 2007;17(1):1-15

9 Sucker C, Litmathe J, Feindt P, Zotz R. Platelet function analyzer (PFA-100) as a useful tool for the prediction of transfusion requirements during aortic valve replacement. Thorac Cardiovasc Surg 2011;59(4):233-236

10 Welsby I, Crow J, Bandarenko N, Lappas G, Phillips-Bute B, StaffordSmith M. A clinical prediction tool to estimate the number of units of red blood cells needed in primary elective coronary artery bypass surgery. Transfusion 2010;50(11):2337-2343

11 Karkouti K, McCluskey SA, Syed S, Pazaratz C, Poonawala H, Crowther MA. The influence of perioperative coagulation status on postoperative blood loss in complex cardiac surgery: a prospective observational study. Anesth Analg 2010;110(6):15331540

12 Wasowicz M, McCluskey SA, Wijeysundera DN, et al. The incremental value of thrombelastography for prediction of excessive blood loss after cardiac surgery: an observational study. Anesth Analg 2010;111(2):331-338

13 Nolan HR, Ramaiah C. Effect of body mass index on postoperative transfusions and 24-hour chest-tube output. Int J Angiol 2011;20 (2):81-86

14 American Heart Association [Internet]. Obesity and overweight. AHA Scientific Position. Updated March 29, 2012. http://www. heart.org/HEARTORG/GettingHealthy/Obesity-and-Overweight_UCM_306016_Article.jsp\#.T1pFKHqi2So. Accessed July 15, 2012

15 Covin R, O’Brien M, Grunwald G, et al. Factors affecting transfusion of fresh frozen plasma, platelets, and red blood cells during elective coronary artery bypass graft surgery. Arch Pathol Lab Med 2003;127(4):415-423

16 Elmistekawy EM, Errett L, Fawzy HF. Predictors of packed red cell transfusion after isolated primary coronary artery bypass grafting -the experience of a single cardiac center: a prospective observational study. J Cardiothorac Surg 2009;4:20
17 Schwann TA, Habib RH, Zacharias A, et al. Effects of body size on operative, intermediate, and long-term outcomes after coronary artery bypass operation. Ann Thorac Surg 2001;71(2):521-530, discussion 530-531

18 Reeves BC, Ascione R, Chamberlain MH, Angelini GD. Effect of body mass index on early outcomes in patients undergoing coronary artery bypass surgery. J Am Coll Cardiol 2003;42(4):668-676

19 Arora RC, Légaré JF, Buth KJ, Sullivan JA, Hirsch GM. Identifying patients at risk of intraoperative and postoperative transfusion in isolated CABG: toward selective conservation strategies. Ann Thorac Surg 2004;78(5):1547-1554

20 Scott BH, Seifert FC, Glass PS, Grimson R. Blood use in patients undergoing coronary artery bypass surgery: impact of cardiopulmonary bypass pump, hematocrit, gender, age, and body weight. Anesth Analg 2003;97(4):958-963

21 van Straten AH, Kats S, Bekker MW, et al. Risk factors for red blood cell transfusion after coronary artery bypass graft surgery. J Cardiothorac Vasc Anesth 2010;24(3):413-417

22 Sensoz Y, Gunay R, Tuygun AK, et al. Computed tomography evaluation of different chest tube sites for residual pleural volumes after coronary artery bypass surgery. Ann Saudi Med 2011;31 (4):383-386

23 Alam M, Siddiqui S, Lee VV, et al. Isolated coronary artery bypass grafting in obese individuals: a propensity matched analysis of outcomes. Circ J 2011;75(6):1378-1385

24 Birkmeyer NJ, Charlesworth DC, Hernandez F, et al; Northern New England Cardiovascular Disease Study Group. Obesity and risk of adverse outcomes associated with coronary artery bypass surgery. Circulation 1998;97(17):1689-1694

25 Kim J, Hammar N, Jakobsson K, Luepker RV, McGovern PG, Ivert T. Obesity and the risk of early and late mortality after coronary artery bypass graft surgery. Am Heart J 2003;146(3):555-560

26 Wynne R, Botti M, Copley D, Bailey M. The normative distribution of chest tube drainage volume after coronary artery bypass grafting. Heart Lung 2007;36(1):35-42

27 Alghamdi AA, Moussa F, Fremes SE. Does the use of preoperative aspirin increase the risk of bleeding in patients undergoing coronary artery bypass grafting surgery? Systematic review and metaanalysis. J Card Surg 2007;22(3):247-256

28 Shapira OM, Aldea GS, Zelingher J, et al. Enhanced blood conservation and improved clinical outcome after valve surgery using heparin-bonded cardiopulmonary bypass circuits. J Card Surg 1996;11(5):307-317

29 Shehata N, Naglie G, Alghamdi AA, et al. Risk factors for red cell transfusion in adults undergoing coronary artery bypass surgery: a systematic review. Vox Sang 2007;93(1):1-11 